\title{
USO DE COBALAMINA NO TRATAMENTO DA NEUROPATIA DIABÉTICA: REVISÃO SISTEMÁTICA DE ENSAIOS CLÍNICOS RANDOMIZADOS
}

\section{USE OF COBALAMIN IN THE TREATMENT OF DIABETIC NEUROPATHY: SYSTEMATIC REVIEW OF RANDOMIZED CLINICAL TRIALS}

\author{
Caio Augusto Carneiro Da Costa'; Benjamin Rodrigues Milagres"; Abraão Lucas Dias Clerot Muniz Paiva';
} Aline Cristina Singulano " Luccas Cavalcanti Garcia $^{\text {Il }}$; Claudia Barros Gonçalves Cunha ${ }^{\text {IV }}$

\begin{abstract}
Resumo. De acordo com a Federação Internacional de Diabetes, estima-se 463 milhões de pessoas acometidas pela doença ao redor do mundo, sendo o Brasil líder de casos na América Latina. Esta revisão objetivou avaliar evidências do uso de vitamina B12 (cobalamina) na melhoria da qualidade de vida de pacientes com Neuropatia Diabética (ND), bem como na redução dos sintomas através da melhora da dor, aumento na percepção sensorial e da avaliação neurológica global. Produziu-se uma Revisão Sistemática de ensaios clínicos randomizados, partindo da seleção e análise de dados, preconizada pela Pubmed, Cochrane e Biblioteca Virtual de Saúde, na qual três revisores selecionaram e extraíram dados, chegando a um consenso entre a qualidade metodológica dos estudos, com objetivo de evitar vieses. Desenvolvido na Faculdade de Medicina Nova Esperança (FAMENE) em parceria da Universidade Federal do Triângulo Mineiro (UFTM), esta pesquisa abrange estudos duplo-cego, placebo controlado, prospectivos, com seguimento e avaliação de pacientes através de escalas e outras avaliações neurológicas. Nesses ensaios, foram avaliados pacientes entre todas as faixas etárias e gêneros, portadores de ND, devidamente diagnosticada, e aptos para realizar reposição oral de vitamina B12. Na primeira fase, foi verificado se cada estudo encontrado cumpria os critérios para inclusão: tipo de estudo, tipo de participantes e tipo de intervenções. Foi identificada a relação estabelecida entre uso de metformina e deficiência de vitamina B12. E, embora existam aumento nos parâmetros neurológicos e escalas avaliadas, não está constatada na literatura ainda a associação benéfica da reposição de cobalamina em pacientes com ND. Portanto, apesar do consenso acerca da deficiência de vitamina B12 causada pelo uso de metformina, ainda não existem evidências científicas referentes à eficiência terapêutica da reposição oral de vitamina B12 em pacientes com diabetes mellitus tipo 2 portadores de ND.
\end{abstract}

PALAVRAS-CHAVE: Diabetes Mellitus Tipo 2. Polineuropatia Diabética. Deficiência de Vitamina B12. Metformina. Neurofisiologia.

Abstract. According to the International Diabetes Federation, an estimated 463 million people are affected by the disease all over the world, Brazil being the leader in cases in Latin America. This article assesses evidence of the use of vitamin B12 in improving the quality of life of patients with Diabetic Neuropathy (DN), as well as reducing its symptoms through pain management, increased sensory perception and global neurological assessment. A Systematic Review of randomized clinical trials was carried out, based on the selection and analysis of data, recommended by PubMed, Cochrane and Biblioteca Virtual de Saúde, in which three reviewers selected, extracted data and come to a consensus about the methodological quality of the studies, with the objective to avoid bias. Developed at the Faculdade de Medicina Nova Esperança (FAMENE), in partnership with the Universidade Federal do Triângulo Mineiro (UFTM), this study encompasses randomized, double-blind, placebocontrolled, prospective clinical trials, containing follow-up and assessment of patients, using scales and further neurological parameters. In these trials, patients of all age groups and genders, with properly diagnosed diabetic neuropathy and able to undergo oral vitamin B12 replacement, were selected. In the first phase, it was assessed whether each study found met the inclusion criteria: type of study, type of participants and type of interventions. A correlation between metformin use and vitamin B12 deficiency was identified and although there is an increase in neurological parameters and evaluated scales, the beneficial association of cobalamin replacement in patients with DN has not yet been established in the literature. Therefore, despite the consensus on vitamin B12 deficiency caused by the use of metformin, there is still no scientific evidence regarding the therapeutic efficiency of oral vitamin B12 replacement in patients with type 2 diabetes mellitus DN associated.

KEYWORDS: Type 2 Diabetes Mellitus. Diabetic Polyneuropathy. B12 Vitamin Deficiency. Metformin. Neurophysiology. 


\section{INTRODUÇÃO}

A Federação Internacional de Diabetes estima que, em 2019, habitavam 463 milhões de pessoas em todo o mundo acometidas por Diabetes Mellitus. No mesmo ano, foi constatado que o Brasil seria o país da América Latina com maior número de diabéticos, com 16,8 milhões de pessoas, ocupando o quinto lugar no mundo. ${ }^{1,2}$

Além disso, há uma grande preocupação quanto a avaliação desses pacientes em todos os níveis de atenção em saúde, tendo em vista a necessidade evidente do acompanhamento regular (Ministério da Saúde e Caderno de controle de Diabetes Mellitus - DM). Além de preocupar-se para desenvolver novas evidências científicas para uma das maiores epidemias do século 21 , o que tem sido o trabalho de muitos pesquisadores brasileiros. $3,4,5,6,7$

A DM2 pode ser causada por resistência insulínica ou pela deficiência das células beta pancreáticas em secretar insulina. A má condição por desordem metabólica resulta em complicações imediatas, como hiperglicemia ou, a longo prazo, em complicações macro e microvasculares. ${ }^{4,8}$ A doença atinge geralmente uma população acima dos 40 anos que representa 90 a $95 \%$ dos casos de DM2; ademais, $80 \%$ dos indivíduos desta classe são obesos, $50 \%$ são assintomáticos e sua maior urgência clínica é a síndrome hiperosmolar hiperglicêmica. ${ }^{7,9}$

Além disso, produtos químicos ou medicamentos também se mostram como causas menores de $\mathrm{DM} 2$, a exemplo dos antipsicóticos atípicos, diuréticos e anti-hiperglicemiantes. Vale ressaltar os principais mecanismos fisiológicos que levam ao quadro hiperglicemiante: resistência periférica a ação insulínica nos adipócitos e no músculo esquelético, deficiência na secreção de insulina pelo pâncreas, aumento da produção hepática de glicose - como resultado da resistência insulínica hepática. Importante lembrar, ainda, os fatores de risco da doença como, por exemplo, a obesidade, hipertrigliceridemia, síndrome metabólica, aumento da circunferência abdominal, diminuição da massa magra e sedentarismo. 4,7

Em se tratando das deficiências de vitamina B12, atualmente existem fortes evidências de que a metformina, fármaco utilizado como tratamento de primeira linha para $\mathrm{DM} 2$, está relacionada a queda dos níveis séricos de vitamina B12 a longo prazo. Esse micronutriente está também intimamente relacionado a composição da bainha de mielina dos axônios, sendo necessária a investigação dos níveis séricos nos pacientes portadores de neuropatias periféricas. ${ }^{10,5}$

Entre as complicações da DM, um grupo de síndromes clínicas causadas pelo dano aos nervos periféricos e autonômicos do sistema nervoso são prevalentes. Estas síndromes são caracterizadas por uma reação local e difusa nos nervos e ocorrem em cerca de $50 \%$ dos pacientes diabéticos. Destes acometimentos, a forma mais comum é a neuropatia periférica distal bilateral. ${ }^{1,2,11}$

Em face disso, faz-se impreterível esta revisão sistemática, a fim de averiguar a relação da vitamina $\mathrm{B} 12$, metformina e neuropatia periférica, além de debater a efetividade da suplementação de vitamina B12 em relação a melhora dos sintomas dos 
quadros neurológicos de pacientes com DM2. Sobre isto, procurou-se discutir a efetividade do tratamento farmacológico na Neuropatia Diabética (ND) quando comparado ao uso de placebo, conduzindo uma revisão sistemática de ensaios clínicos randomizados. O objetivo do

\section{MATERIAL E MÉTODOS}

$\begin{array}{ccc}\text { Foi realizada uma Revisão } & \\ \text { Sistemática de ensaios clínicos }\end{array}$ randomizados, localizados nas bibliotecas de pesquisa virtual, durante o ano de 2021, desenvolvida na Faculdade de Medicina Nova Esperança (FAMENE), Departamento de Neuroanatomia, em parceria com a Universidade Federal do Triângulo Mineiro (UFTM).

Foram selecionados ensaios clínicos randomizados, somente em humanos, realizados nos últimos 10 anos do tipo duplo-cego e placebo controlado, em inglês, português, que avaliam a efetividade da vitamina B12 na Neuropatia Diabética (ND); todas as idades e ambos os sexos foram incluídos. Estudos que não avaliaram a efetividade da vitamina B12 na ND foram incluídos ou excluídos de acordo com a seleção dos autores e revisados através da leitura e posterior discussão aos pares.

Todas as buscas foram conduzidas para trabalhos indexados, publicados entre 2011 e 2021. As seguintes bases de dados foram utilizadas: PubMed (20112021), Registro de Ensaios Controlados da Cochrane (2020), e BVS (2011-2021), para identificação de ensaios clínicos randomizados. Para essas bases de dados específicas foi utilizada a estratégia de busca padronizada para identificação de ensaios clínicos randomizados, associados estudo foi averiguar a efetividade da terapêutica da reposição de vitamina B12 (metilcobalamina), com pelo menos 1000 ug/dia, através do aumento na velocidade de condução nervosa, da melhora da dor neuropática e da percepção sensorial em pacientes com ND. às palavras-chave, ou MeSH-terms desta revisão.

Foram selecionados os seguintes termos para busca: randomized controlled trial [Publication Type] OR controlled clinical trial [Publication Type] OR randomized controlled trials [MeSH Terms] OR random allocation [MeSH Terms] OR double blind method [MeSH Term.s] [Text Word] OR double* [Text Word] OR treble* [Text Word] OR triple* [Text Word] OR placebos [MeSH Terms] OR placebo* [Text Word] OR random* [Text Word] OR research design [MeSH Terms] OR comparative study [MeSH Terms] OR evaluation studies [MeSH Terms] OR follow-up studies [MeSH Terms].

Ainda, a seleção dos estudos foi feita de forma independente, observandose a pergunta da pesquisa e do desenho metodológico estudado. A seleção de estudos foi conduzida por três revisores.

O contato com os autores dos trabalhos para esclarecimentos sobre dados $e$ metodologia foi limitado. Seguindo a metodologia da Revisão Sistemática da Cochrane, os trabalhos foram avaliados em três momentos, explicados abaixo:

$\mathrm{Na}$ primeira fase foi verificado se cada estudo encontrado cumpre os critérios para inclusão: ensaios clínicos randomizados, tipo de participantes 
e tipo de intervenções. Desse modo, os dados foram independentemente ordenados pelos revisores e incorporados a uma base de dados computadorizada (Mendeley e RevMan). Em um segundo momento, os dados foram cruzados pelos três revisores. A coleta dos dados foi a mais precisa possível, com o máximo de rigor na busca dos detalhes, procurando, inclusive, usar exatamente as mesmas palavras empregadas pelos autores na descrição de cada item. No software Mendeley, acrescentaram-se as referências

\section{RESULTADOS E DISCUSSÃO}

Foram encontrados doze ensaios clínicos, através das palavras-chave, e um deles foi excluído por não se enquadrar nos critérios metodológicos.

Em estudo realizado, comparando a prevalência de deficiência de vitamina B12 e Neuropatia Diabética (ND) entre dois grupos de pacientes portadores de DM2, verificouse que o uso de Metformina em dosagens maiores que $2.000 \mathrm{mg}$ por dia e por mais de 4 anos está associado a deficiência de vitamina B12. No entanto, não apresentou correlação estatística significante com a incidência de neuropatia periférica. ${ }^{12,13}$

Uma revisão sistemática ${ }^{14}$ realizada em 2014 e publicada no Singapore Medical Journal, concluiu que a vitamina B12 (isolada ou em combinações terapêuticas) não é candidata provável para o tratamento de ND devido à ineficiência em reduzir sintomas e melhorar parâmetros eletrofisiológicos. Além disso, outro estudo avaliado por Jayabalan e Low ${ }^{15}$ relata que, mesmo a vitamina B12, sendo uma terapia segura e com mínimos efeitos adversos, não há evidência para para padronização e sistematização das informações necessárias. Elaborou-se o projeto do trabalho em software: RevMan, a fim de facilitar a organização da revisão. Depois de ler e avaliar os dados, em um terceiro momento, foi possível comparar os estudos entre si, a fim de verificar semelhanças e diferenças. A qualidade foi avaliada pelos componentes que foram coletados em quatro seções (método, participantes, intervenção e desfechos clínicos).

suplementação oral de vitamina B12.

Em relato de caso realizado por Aaron e Vinik ${ }^{16}$, publicado na New England Journal of Medicine, no ano de 2016, sobre o manejo de um paciente com sessenta e cinco anos, diagnosticado com diabetes mellitus há 5 anos, foi verificada forte associação entre deficiência de B12 e uso de metformina, prejudicando a percepção da sensibilidade vibratória e perda dos reflexos do tornozelo.

Em ensaio clínico randomizado (RCT) de 4,3 anos, demonstrou que a metformina leva à deficiência de vitamina B12 de forma dependente à dose e duração do tratamento. ${ }^{12,17}$ Foi evidenciado ainda que a metformina reduziu, em cerca de $30 \%$ dos pacientes, a absorção de vitamina B12 no íleo terminal, podendo surgir a partir de 4 meses de início do uso. A deficiência de cobalamina geralmente surge de 4 a 5 anos após o uso do anti-hiperglicemiante. ${ }^{2,18}$

Em um ensaio, buscou-se avaliar, através de um estudo duplo-cego, randomizado, placebo controlado $(n=90)$, em pacientes portadores de DM2 com neuropatia 
periférica, o efeito da suplementação oral de vitamina B12 (metilcobalamina) $1000 \mathrm{mg}$ por dia por um período de 1 ano (todos os pacientes tinham níveis de vitamina B12 menores que $400 \mathrm{pmol} / \mathrm{L})$. Os indivíduos foram divididos em 2 grupos, sendo o grupo controle $(n=46)$ que recebeu placebo, e o grupo em tratamento ativo $(n=44)$ que recebeu a metilcobalamina. Os grupos passaram por avaliações para quantificação de níveis de B12 sérico, variáveis neurofisiológicas e funcionais, assim como questionários para avaliação de qualidade de vida e dor. Todos os pacientes tomaram metformina por pelo menos 4 anos, nenhum dos pacientes estavam em tratamento de insulina basal diária. Não houve diferença significativa entre grupo ativo e grupo placebo referente a variáveis antropométricas, demográficas, laboratoriais e testes neurológicos. A sensibilidade vibratória avaliada pelo exame físico neurológico, o Michigan neuropathy screening instrument - questionnaire portion (MNSIQ), a qualidade de vida, questionários sobre dor, velocidade de condução e potencial de ação do nervo sural, bem como a condução eletroquímica da pele foram avaliados nos grupos em questão. Foi visto um aumento significativo no grupo ativo com relevância estatística quando comparado ao grupo placebo. ${ }^{12,13}$

Além disso, em estudo clínico realizado por Mottaghi et al ${ }^{14}$ entre 2017 e 2018, duplo-cego, randomizado, comparando dois grupos com suplementação 1000 $m g$ ácido fólico $(n=40) \times$ placebo $(n=40)$ foi avaliada a velocidade de condução do estímulo sensorial durante 16 semanas. Foi visto o aumento na velocidade de condução e o aumento da amplitude sensorial e motora do nervo sural no grupo ativo $(p<0,001)$. No entanto, houve diminuição no tempo latência da condução do estímulo motor dos nervos perineais $(p<0.0019)$ e tibial $(p<0.011)$. Neste estudo, foi concluído que os componentes da velocidade de condução nervosa são significativamente aumentados pela suplementação de ácido fólico, mas não atingem os níveis considerados normais para população não diabética.

Outrossim, em estudo chinês multicêntrico, randomizado, duplo-cego, placebo controlado: em fase 2, foi avaliada a utilização de I-carnitina $500 \mathrm{mg} \quad(\mathrm{n}=117)$ comparada com $0,5 \mathrm{mg}$ metilcobalamina $(n=115)$ por 24 sem. Após esse período, foi relatada melhora nos sintomas neuropáticos, escalas de funcionalidade e parâmetros fisiológicos nos dois grupos, sem grandes diferenças estatísticas entre as duas populações, exceto pela velocidade de condução motora do nervo ulnar que foi significativamente maior no grupo da metilcobalamina. $^{19}$

Foi avaliada ainda uma revisão sistemática ${ }^{20}$, contendo metanálise de ensaios clínicos randomizados para verificar eficácia e segurança da terapia com vitamina B12 em portadores de ND. Sendo avaliada a escala de dor, sintomas, velocidade de condução nervosa e efeitos adversos. Ao todo, quinze estudos foram avaliados $(n=1707)$. Dentre os grupos avaliados, tanto a metilcobalamina isolada (risk ratio [RR] = 1.17; 95\% confidence interval $[\mathrm{Cl}] 1.03-1.33$ ) como a metilcobalamina combinada com outras moléculas ( $R R=1.32 ; 95 \% \mathrm{Cl} 1.21-1.45)$ foram mais eficazes que o grupo controle.

Entre os anos de 2008 e 2013 foi realizado um ensaio populacional israelense com 5131 pacientes com DM2 que não faziam tratamento para doença e iniciaram a metformina no período em questão, por pelo menos 6 meses. Investigou-se a prática médica e os determinantes clínicos de teste de vitamina $\mathrm{B} 12$ em pacientes com $\mathrm{DM} 2$ tratados com metformina e apenas $45 \%$ dos participantes receberam indicação de fazer 
testes de vitamina B12, evidenciando falta de atuação clínica. $^{21}$

Avaliou-se uma meta-análise de ensaios clínicos randomizados sobre pacientes com ND que realizaram terapia combinada de vitamina B12 e prostaglandina E1. Entre os grupos, tanto a terapia combinada quanto a monoterapia apresentaram melhora significativa dos sintomas clínicos e velocidade de condução nervosa. Quando comparados entre si, a terapia combinada se demonstrou superior em promover melhora dos parâmetros avaliados. ${ }^{22}$ Ao fim do levantamento de dados, chama a atenção o número bastante restrito de estudos que avaliem as variáveis de interesse e suas correlações, ficando limitada a evidência disponível para a interpretação e aplicação clínica dos resultados.

Atualmente, para controle e combate desta doença, a vitamina B12 tem-se mostrado como a principal suplementação terapêutica, exibindo bons resultados associada a um bom controle glicêmico. A deficiência de vitamina B12 é significativa e presente em pacientes com DM2, podendo alcançar 50\% dos indivíduos portadores, sendo essa a mesma porcentagem de pacientes portadores de DM2 associada a ND.3,17 Ademais, o quadro clínico causado pela deficiência de vitamina B12 caracteriza-se por distúrbios de ordem neurológica, podendo apresentar características periféricas, autonômicas (incluindo de ordens cardiovasculares) e neuropatias dolorosas, caracterizando um quadro similar ou acelerar a progressão da ND.18 Reforça-se ainda, a ocorrência da deficiência de vitamina B12 com escassez de características hematológicas típicas, como pancitopenia ou anemia megaloblástica. ${ }^{9}$

Segundo a American Diabetes Association (ADA), é recomendado que pacientes com ND, em terapia regular com metformina, tenham os níveis de cobalamina supervisionados de maneira regular em uma base anual. ${ }^{2}$ Vale ressaltar que, para o rastreio em pacientes portadores de DM2 com idade superior à 60 anos, os valores de referência para a deficiência de vitamina B12, capazes de acarretar uma disfunção neurológica sejam movidos de 150 para 400 pmol/L, com prevalência confirmada desta deficiência em 12 a $23 \%$ dos indivíduos. ${ }^{16,23}$

Sendo assim, foi visto que pacientes portadores de DM2, tratados com metformina, comprovadamente possuem déficit nos níveis séricos de vitamina B12. Além disso, foi identificada uma menor probabilidade de se solicitar testes sanguíneos em indivíduos acima de 75 anos, justificado por uma ineficiência da prática geriátrica e negligência com os idosos. Outrossim, os estudos demonstraram que a prevalência de deficiência de vitamina B12 chega a atingir $40 \%$ dos pacientes da população acima de 50 anos, o que sugere fortemente a existência de subdiagnóstico da deficiência de vitamina B12. ${ }^{21}$

No entanto, ensaios clínicos randomizados nos quais se analisou pequenas populações, durante um breve período de acompanhamento, tiveram como resultado a ausência de associação entre metformina, deficiência vitamina B12 e neuropatia periférica. Sabe-se que amostras pequenas influenciam negativamente na confiabilidade dos dados, sendo necessários mais estudos e de maior qualidade, pois foram evidenciadas divergências nos resultados, sugestivos de vieses de aferição. ${ }^{17}$

Em relação às terapêuticas avaliadas, foi visto que tanto o uso de l-carnitina, como da vitamina B12 promoveram significativa melhora da sensibilidade vibratória, avaliada pelo exame físico neurológico, da qualidade de vida, e da velocidade de condução e potencial de ação do nervo sural, bem como da condução eletroquímica da pele 
nos grupos em questão. Essa análise traz à tona uma reflexão sobre a associação da neuropatia e a deficiência de vitamina B12, além da possibilidade de sinergismo de ação das medicações em questão. ${ }^{12,13,14,15,22}$

Destarte, resta saber até que ponto a neuropatia periférica estaria associada à vitamina B12. Seria válida a suplementação profilática de tal vitamina? Até que ponto esse subdiagnóstico pode atrapalhar no manejo desses pacientes e quais outros fatores podem estar associados a neuropatia periférica.

\section{CONSIDERAÇÕES FINAIS}

É conhecido o potencial da metformina em reduzir os níveis séricos de vitamina B12, assim como seu uso enquanto primeira opção terapêutica entre os fármacos anti-hiperglicemiantes para tratamentos dessa população.

A relação entre metformina e a deficiência de cobalamina é pouco valorizada na prática clínica apesar de sua comprovada relação. Devendo-se incentivar a investigação de tal deficiência e incluir sua pesquisa na rotina dos pacientes com $\mathrm{DM} 2$ e portadores de ND.

Estabelece-se ainda a recomendação pela triagem de deficiência de vitamina B12 em paciente acima de 50 anos, portadores de diabetes mellitus tipo 2 e que fazem uso de metformina, devido a prevalência da deficiência em até $40 \%$ dos indivíduos neste grupo.

Aliás, trazendo à tona a necessidade de elucidar a relação entre metforminavitamina B12 e neuropatia periférica faz-se necessária a realização de novos estudos e trabalhos que avaliem grandes bancos de dados de registros eletrônicos e utilizem ferramentas de avaliação mais objetivas a fim de estabelecer melhores parâmetros quanto à significância clínica da redução sérica e sua relação com os níveis celulares de vitamina B12.
Destarte, quanto à profilaxia, não se tem recomendação formal fazendo-se necessária a realização de novos ensaios de boa qualidade metodológica para verificar a eficácia da profilaxia em pacientes portadores de diabetes mellitus associadas à ND capazes de avaliar as possíveis qualidades adjuvantes da terapia associada à vitamina B12.

No que se refere ao tratamento, há benefício com dose terapêutica mínima de 1.00omg de vitamina B12 em distintas apresentações, para pacientes com níveis séricos menores que $400 \mathrm{pmol} / \mathrm{L}$.

Desse modo, o uso de vitamina B12 ainda permanece controverso, pois embora demonstre melhora dos parâmetros neurofisiológicos e das escalas de avaliação subjetivas, a percepção de sensibilidade, motricidade e condutibilidade ainda permanecem baixas.

Portanto, outras medicações que possam apresentar mecanismos de ações diferentes na reconstituição da bainha de mielina, como também de moléculas que possam inibir as lesões microangiopáticas causadas pela diabetes precisam ser investigadas. Ademais, novos estudos avaliando o benefício dessas substâncias e a possível melhora dos parâmetros devem ser investigadas em comparação à população não-diabética. 


\section{REFERÊNCIAS BIBLIOGRÁFICAS}

1. Feldman EL, Callaghan BC, Pop-Busui R, Zochodne DW, Wright DE, Bennett DL, etal. Diabetic neuropathy. Nat Rev Dis Primers. 2019; 5(1): 41.

2. International Diabetes Federation. IDF Diabetes Atlas, 10th edn. Brussels, Belgium: International Diabetes Federation, 2021.

3. Faludi AA, Izar MC de O, Saraiva JFK, Bianco HT, Chacra APM, Bertoluci MC, et al. Diretriz brasileira baseada em evidências sobre prevenção de doenças cardiovasculares em pacientes com diabetes: Posicionamento da sociedade brasileira de diabetes (SBD), da sociedade brasileira de cardiologia (SBC) e da sociedade brasileira de endocrinologia, Arq Bras Cardiol. 2017; 109(6Supl.1): 1-31.

4. Hovadick, Ana Carolina de Andrade, Reis, Ilka Afonso e Torres, Heloísa Carvalho.Short Message Service (SMS) e promoção do autocuidado em DM2: revisão integrativa. Acta Paulista de Enfermagem. 2019; 32(2): 210-19

5. SBD - Sociedade Brasileira de Diabetes. Diretrizes da Sociedade Brasileira de Diabetes 2019-2020. 2019: 419.

6. Souza CF, Gross JL, Gerchman F, Leitão CB. Prédiabetes: Diagnóstico, avaliação de complicações crônicas e tratamento. Arq Bras Endocrinol Metabol. 2012; 56(5): 275-84.

7. Vilar L, Lyra R, Azevedo Junior LGG, Diniz ET, Ibiapina GR, Veloso IGL, Frasão $\mathrm{K}$ et al. Diabetes melito. Endocrinologia clínica. 2016; 6: 641-56.

8. Alharbi TJ, Tourkmani AM, Abdelhay O, Alkhashan $\mathrm{HI}$, Al-Asmari AK, Bin Rsheed AM, et al. The association of metformin use with vitamin B12 deficiency and peripheral neuropathy in Saudi individuals with type 2 diabetes mellitus. PLoS ONE, 2018; 13(10): 1-15.

9. Ahmed, M. A. Metformin and Vitamin B12 Deficiency: Where Do We Stand?. J. Pharm. Pharm. Sci., 2016; 19(3): 382-98.
10. Giménez Roldán S. Tratado de neurología. Rev Neurol. 2012; 54(8): 512.

11. Callaghan BC, Price RS, Chen KS, Feldman EL. The Importance of Rare Subtypes in Diagnosis and Treatment of Peripheral Neuropathy: A Review. JAMA Neurol. 2015; 72(12): 1510-8.

12. Didangelos T, Karlafti E, Kotzakioulafi E, Margariti E, Giannoulaki P, Batanis G, el al. Vitamin B12 Supplementation in Diabetic Neuropathy: A 1-Year, Randomized, Double-Blind, Placebo-Controlled Trial. Nutrients. 2021; 13(2): 395.

13. Didangelos T, Karlafti E, KotzakioulafiE, Kontoninas Z, Margaritidis C, Giannoulaki P, et al. Efficacy and Safety of the Combination of Superoxide Dismutase, Alpha Lipoic Acid, Vitamin B12, and Carnitine for 12 Months in Patients with Diabetic Neuropathy. Nutrients. 2020; 12(11): 3254.

14. Mottaghi T, Khorvash F, Maracy M, Bellissimo $\mathrm{N}$, Askari G. Effect of folic acid supplementation on nerve conduction velocity in diabetic polyneuropathy patients. Neurol Res. 2019; 41(4): 364-68.

15. Jayabalan B, Low LL. Vitamin B supplementation for diabetic peripheral neuropathy. Singapore Med J. 2016;57(2):55-9.

16. Aaron I. Vinik, Diabetic Sensory and Motor Neuropathy, N. Engl. J. Med., 2016;374(15): 1455-464. 17. Donato $\mathrm{H}$, Donato $\mathrm{M}$. Stages for undertaking a systematic review. Acta Médica Portuguesa. 2019; 32(3): $227-35$.

18. Hansen CS, Jensen JS, Ridderstråle $M$, Vistisen $D$, Jørgensen ME, Fleischer J. Vitamin B12 deficiency is associated with cardiovascular autonomic neuropathy in patients with type 2 diabetes. J Diabetes Complications. 2017; (1): 202-08.

19. Li S, Chen X, Li Q, Du J, Liu Z, Peng Y, et al. Effects of acetyl-L-carnitine and methylcobalamin for diabetic peripheral neuropathy: A multicenter, randomized, 
double-blind, controlled trial. J Diabetes Investig. 2016; 7(5): 777-85.

20. Sawangjit R, Thongphui S, Chaichompu W, Phumart P. Efficacy and Safety of Mecobalamin on Peripheral Neuropathy: A Systematic Review and Meta-Analysis of Randomized Controlled Trials. J Altern Complement Med. 2020; 26(12): 1117-1129.

21. Fogelman Y, Kitai E, Blumberg G, Golan-Cohen A, Rapoport M, Carmeli E. Vitamin B12 screening in metformin-treated diabetics in primary care: were elderly patients less likely to be tested? Aging Clin
Exp Res. 2017; 29(2): 135-39.

22. Wang $Y$, Jiang DQ, Zhao SH, Li MX, Jiang LL, Wang Y. Prostaglandin E1 plus methylcobalamin combination therapy versus prostaglandin E1 monotherapy for patients with diabetic peripheral neuropathy. Med (United States). 2018; 97(44): 1-8.

23. Beulens J.W., Hart H.E., Kuijs R., Kooijman-Buiting A.M.J., Rutten G.E.H.M. Influence of duration and dose of metformin on cobalamin deficiency in type 2 diabetes patients using metformin. Acta Diabetol. 2015; 52(1): 47-53. 